\section{(6) OPEN ACCESS}

${ }^{1}$ Department of Global Public Health and Primary Care, Global Health Priorities, University of Bergen, Bergen, Norway ${ }^{2}$ Health Services Research Unit (HØKH), Akershus University Hospital, Lørenskog, Norway

\section{Correspondence to} Dr Carl Tollef Solberg, Department of Global Public Health and Primary Care, Global Health Priorities, University of Bergen, Postboks 7804, Bergen N-5020, Norway; carl.solberg@uib.no

Received 9 May 2017 Revised 28 July 2017 Accepted 24 September 2017 Published Online First 27 October 2017 medethics-2017-104635

Check for updates

To cite: Solberg $\mathrm{CT}$, Norheim OF, Barra M. $J$ Med Ethics

2018:44:192-198

\title{
The disvalue of death in the global burden of disease
}

\author{
Carl Tollef Solberg, ${ }^{1}$ Ole Frithjof Norheim, ${ }^{1}$ Mathias Barra ${ }^{2}$
}

\section{ABSTRACT}

In the Global Burden of Disease study, disease burden is measured as disability-adjusted life years (DALYs). The paramount assumption of the DALY is that it makes sense to aggregate years lived with disability (YLDs) and years of life lost (YLLS). However, this is not smooth sailing. Whereas morbidity (YLD) is something that happens to an individual, loss of life itself (YLL) occurs when that individual's life has ended. YLLs quantify something that involves no experience and does not take place among living individuals. This casts doubt on whether the YLL is an individual burden at all. If not, then YLDs and YLLs are incommensurable. There are at least three responses to this problem, only one of which is tenable: a counterfactual account of harm. Taking this strategy necessitates a re-examination of how we count YLLs, particularly at the beginning of life.

\section{THE GLOBAL BURDEN OF DISEASE}

The Global Burden of Disease (GBD) is 'a systematic, scientific effort to quantify the comparative magnitude of health loss due to diseases, injuries and risk factors by age, sex and geographies for specific points in time' (Murray et al, p2063 ${ }^{1}$; Mu rray et $a l, \mathrm{p} 1^{2}$ ). Since its first publication in the early 1990s, the GBD has grown to be a worldwide endeavour drawing on the expertise of several principal actors in global health. ${ }^{3}$ Historically, epidemiologists have concerned themselves chiefly with the mortality in a population and mortality rates and deaths averted were the standards for measuring disease burden. ${ }^{45}$ The GBD, however, aims to integrate morbidity and mortality into disability-adjusted life years (DALYs). This summary measure is a significant contribution to studies of global health, as mortality alone cannot adequately capture what is intuitively understood by 'the burden of disease'. To measure disease burden, we need to take account of both morbidity and mortality.

The DALY metric-the aggregation of years lived with disability (YLDs) and years of life lost (YLLs)- has become popular in the global health community. Publications using DALYs regularly feature in top journals, like The Lancet and the New England Journal of Medicine, ${ }^{136}$ and major organisations, like WHO and the World Bank, ${ }^{7}$ use DALY estimates. Furthermore, the GBD studywhich uses the DALY as its health metric-is used as evidence for informed policy-making. ${ }^{8}$ Yet, if the DALY is not conceptually sound, we risk ranking disabilities wrongly, measuring inequality in health on false premises, and perhaps even ranking health programmes suboptimally.

It is, therefore, vital that all the assumptions underlying the DALY are as well founded, coherent and plausible as possible. The DALY model relies on several assumptions that have been revised over the years. ${ }^{2}$ Previous operationalisations of the DALY included age-weighting, time discounting, sex differentiation and the use of Japanese life tables as a yardstick for an ideal lifespan to compute YLLs. Current practices, such as involving the public in setting disability weights (DWs) for the calculation of YLDs or using synthetic life tables across the globe for estimating the YLL at the time of death, reflect changing assumptions and recognition of the potential for improvement in the measure. ${ }^{8-12}$

Nonetheless, there is at least one critical assumption that, we maintain, has not been given enough attention: the assumption that it makes sense to aggregate YLLs with YLDs into DALYs. ${ }^{\mathrm{i}}$ The aim of this paper is to address that underlying assumption.

\section{THE DALY MODEL}

The DALY model was developed during the late 1980s and early 1990s, building on earlier timebased measures for quantifying health. ${ }^{451314}$ The GBD 1990 launched the DALY, and its raison d'être was to obtain an integrated measure of morbidity and mortality. Since its conception, the DALY has primarily been concerned with individual burden. However, the need to clarify how death can be an individual burden may have been veiled from the first DALY model as this included age-weighting to reflect the '[s] ocial [burden] of the time lived at different ages' (Murray, pp434-436) ${ }^{15}$ Following a methodological debate during the ensuing decades, societal burden has been excluded. The most recent DALY model has been revised with the explicit aim to reflect the individual's own burden of disease, exclusively. ${ }^{\text {ii2 }}$ Accordingly, the DALY needs to incorporate a well-founded account of the individual burden of death which sets this paper's topic at centre stage.

Today, DALYs measure the unhealth in a population understood as aggregated individual burden. ${ }^{1}$ Unlike most other time-based health-outcome measures-such as the quality-adjusted life year (QALY), which tries to put a value on the time spent in various (health) states ${ }^{16}$ - the DALY purports to measure health impairment directly.

Some will suggest that our critique of the DALY will affect the QALY to an equal degree, partly because of the common misconception that 'DALYs

${ }^{\mathrm{i}}$ This problem should not be confused with the incidence versus prevalence perspective for DALYs. ${ }^{8} 153545$

${ }^{\text {ii } I n ~ t h e ~ w o r d s ~ o f ~ M u r r a y ~ e t ~ a l ~ ' b u r d e n ~ s h o u l d ~ b e ~ a s s e s s e d ~}$ individual by individual' (p14). ${ }^{2}$ However, the numerous value assumptions within the DALY make it doubtful that this aim has been achieved. ${ }^{9-124647}$ 
Panel 1: Visualisation of the QALY as an area under the curve

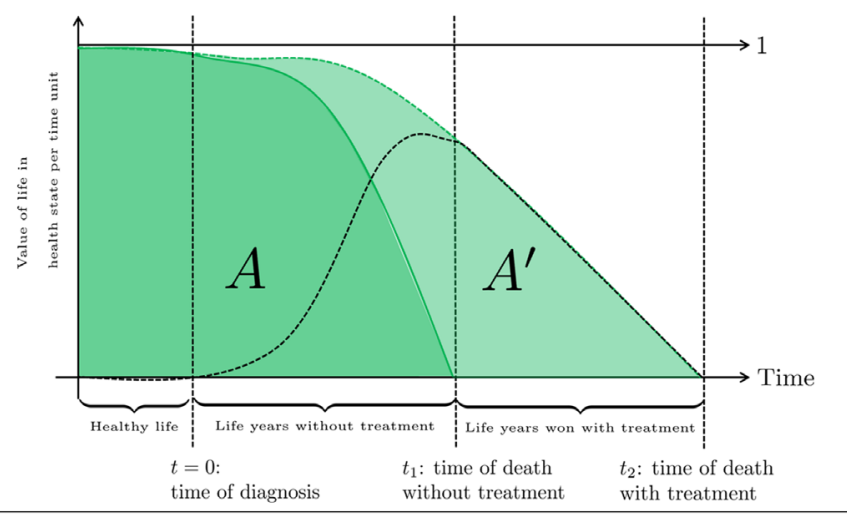

Panel 2: QALYs gained in the two scenarios:

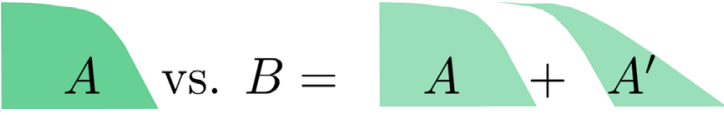

Figure 1 The quality-adjusted life year (QALY) model. Figure 1 illustrates the QALY for one individual. In panel 1 the leftmost dashed vertical line represents the time of diagnosis and a possibility for starting up with treatment. This is followed by one of two counterfactual scenarios: One scenario is illustrated by the solid green curve (we refer to this as $h_{1}$ ) and represents the likely progression without treatment. This scenario results in a number of experienced QALYs that is equal to the area under the curve (AUC) denoted $A$ (see panel 2). The alternative scenario is illustrated by the dashed green line (we refer to this as $h_{2}$ ) and represents the likely progression with treatment. In this alternative, the QALYs experienced are represented by the AUC denoted $B=A+A^{\prime}$ (see panel 2). The difference in accrued QALYs between these two counterfactual scenarios is the QALY gain, here represented by the area $A^{\prime}$. In order to calculate $A^{\prime}$ we need not rely on the assumption that the value of 'being dead' is 0 . We see that the QALY gain $A^{\prime}$ can be computed as the difference between the two areas: $A^{\prime}=B-A$. However, if one would like (as is often convenient) to compute this difference as the integral between two curves - viz as roughly the area beneath the dashed black curve-then the scenario without treatment must be equipped with a value $u_{\text {death }}$ for the part of the integral where only $h_{2}$ is initially defined; as $h_{1}$ is a priori only defined between 0 and $t_{1}$. In order to be consistent with the QALY paradigm $u_{\text {death }}$ must satisfy the equation: $\int_{0}^{t_{2}} h_{2}(x) d x-\int_{0}^{t_{1}} h_{1}(x) d x=\int_{0}^{t_{1}} h_{2}(x)-h_{1}(x) d x+\int_{t_{1}}^{t_{2}} h_{2}(x)-u_{\text {death }} d x$. This equation obviously obtains if and only if $u_{\text {death }}=0$.

are inverse QALYs'. ${ }^{17-19}$ In fact, the DALY differs fundamentally from the QALY in at least two important respects. First, the DALY is a so-called negative gap-measure purporting to quantify the burden suffered as a consequence of experienced unhealth directly. $^{2815}$ In contrast, the QALY measures the value of being alive. Second, while the QALY does not require any particular value associated with 'being dead', the DALY is defined in terms of such a burden. In the words of Murray and colleagues: '[t]he basic unit of measurement for [the DALY] is lost years of healthy life' (p14). ${ }^{2}$ Taken together, the DALY thus assumes and relies on a coherent concept of the individual burden associated with 'being dead'. The QALY model does not. The QALY is a pure time-based measure of the value of time spent alive relative to one's health state.
While it is common to describe the QALY model as assigning the zero value to 'being dead', ${ }^{16}$ this is merely a heuristic. In fact, the QALY assigns, in the limit, zero value as time approaches zero which is what endows the QALY model with ratio scale properties, rather than just interval scale properties. ${ }^{20}$ The primary attribute of death in the QALY model is that for the dead, time is no more; accordingly, there is nothing to be valued. We can however extend the QALY model by assigning zero to 'being dead'. In fact, this is the only consistent extension of the QALY model, compatible with the necessary assumption that the dead are expelled from the matrix of time.

To sum up, the QALY is concerned with living individuals exclusively, and nothing in the QALY model corresponds to the YLL. When an individual die, intraindividual QALY aggregation ends. In the DALY model, YLLs-which does not happen during your life-are integral. The estimation of QALY values requires comparing various durations of time spent in different health states, and never relies on any direct comparisons with death. The DALY, on the other extreme, postulates that a year of 'being dead' is a viable unit for burden. While the QALY losses estimated for a deceased individual may be said to assume something about the non-aggregation of QALYs after death, this is, in fact, not part of the QALY model itself. In the DALY model, the burden of death is interwoven into its fabric. This latter observation contradicts the widely held belief that DALYs are inverse QALYs, and suggests that this heuristic for understanding one of these models in terms of the other has been extrapolated too far. While the QALY resides among the living, the DALY model must cross the river Styx.

See figure 1 for a graphical illustration. For the DALY, as we argue below, the situation is entirely different.

\section{YEARS OF LIFE LOST}

The fundamental principle that ' $[\mathrm{t}]$ ime is the unit of measure for the burden of disease' has been retained from the original formulation of the DALY (Murray et al, p132; Murray, p4312). The DALY component the YLL is by definition taken as a burden. It is assumed to be quantified by itself: the number of years in the gap between the time of an individual's death and some ideal length of life is defined as identical to the burden imposed on that individual by his/her death. There are many issues pertaining to estimating the ideal remaining lifespan of an individual at the time of his/her premature death. However, the previous paragraph covers the fundamental definition of the YLL: burden equals the number of life years lost.

\section{YEARS LIVED WITH DISABILITY}

To integrate mortality-measured by the YLL-and morbidity, the DALY model includes a second component: the YLD. In order to define the YLD, time is again taken as the basic unit of measurement. ${ }^{2}$ Previous works on the YLD have relied on QALYtype measurements, including standard gamble, time trade-off and person trade-off. ${ }^{1521}$ Furthermore, in line with the explicit aim of not measuring disvalue as such, but rather 'objective unhealth', the current YLD relies on a representative sample of laymen's evaluation of the degree of unhealth represented by various medical conditions, suitably described by an expert panel. The DWs are then assigned based on a mixture of two methods: the person trade-off and latent utility-like discrete choice tasks. ${ }^{2}$

This procedure results in DWs $\left(D W_{A}\right)$ for the various conditions $A$, which yield YLDs when multiplied by duration $T$ :

$$
\mathrm{DW}_{A} \times T=\mathrm{YLD}
$$




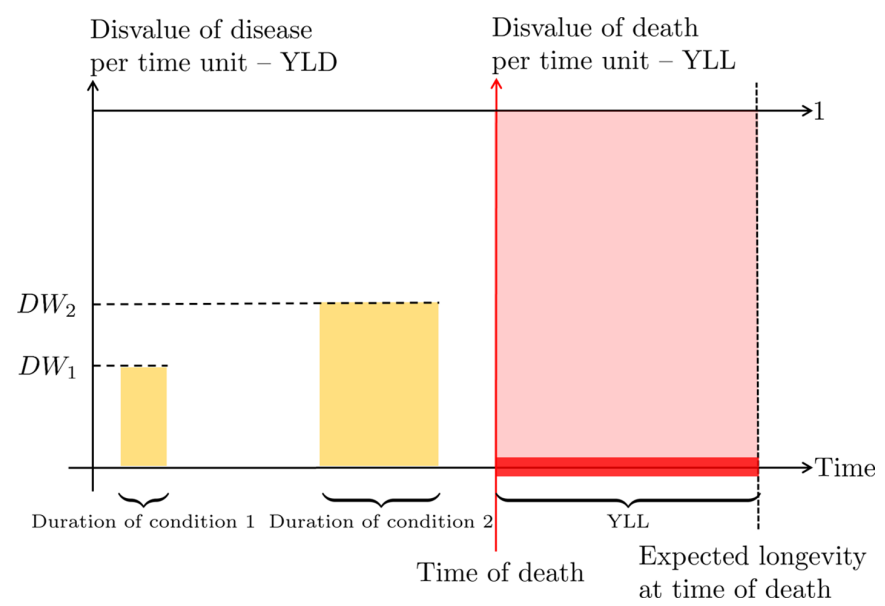

Figure 2 The DALY model. Lifetime DALYs for an individual with two periods of illness, followed by premature death. The yellow and red colours represent YLDs and YLLs, respectively. The YLD areas are delineated by the duration on the time axis and the disability weights $D W_{\mathrm{i}}$ for the two conditions. The light red area is defined by erecting a rectangle of height 1 over the dark red line segment representing the number of years of life lost to premature death. The total of the light red and yellow areas constitutes the DALY. The DALY model assumes that it makes sense to attribute a burden of 1 to the YLLs; we challenge this assumption. DALYs, disability-adjusted life years; YLDs, years lived with disability; YLLs, years of life lost.

The DALYs are then computed as the sum of the YLDs and the YLLs (see figure 2).

But note how the equation reduces to 0 if time is zero. This equation hints as to what we view as the problem with the current DALY: the YLD is a measure of an inherently two-dimensional

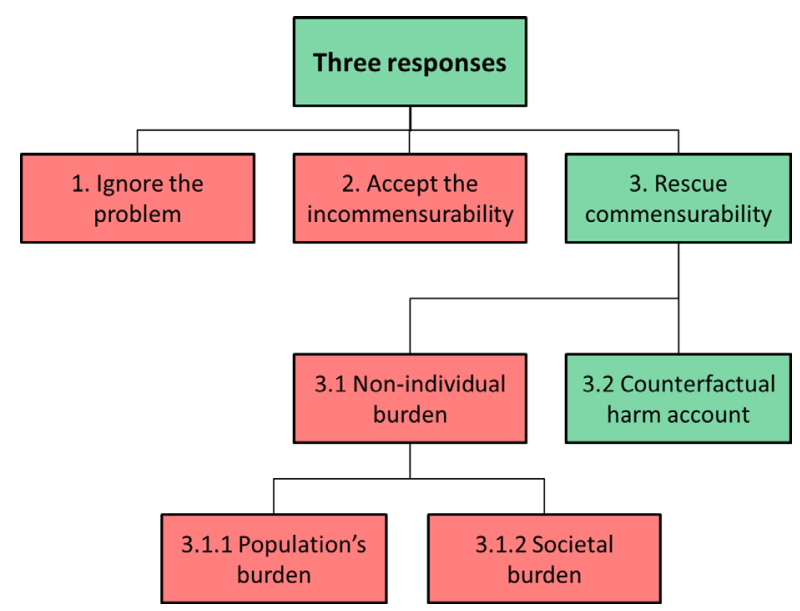

Figure 3 Three responses. We explore three possible responses to the incommensurability problem, two of which we consider untenable (1 and 2). On the rescue strategy (3) there are two main avenues: (3.1) argues that YLDs and YLLs, in fact, quantify something other than individual burden, while (3.2) (which we endorse) argues that the YLD and the YLL can be commensurableas individual burden-conditional on adopting a counterfactual account of harm. YLDs, years lived with disability; YLLs, years of life lost. construct with unit $D W \times T$-burden of disability times duration. The YLL, on the other hand, is by definition a one-dimensional construct, measured by its duration only. Unless a sound DW for 'being dead' can be produced, one cannot add YLLs and YLDs together in its current form. The reason is that the first has unit time, while the latter has unit disability times time. An analogy would be adding a length to an area.

There are at least two objections to this dimensional concern. First, some may maintain that the DWs are mere scalars which means that the YLD measures a percentage of the YLL. Then, being blind $(\mathrm{DW}=0.19$ : this and later $\mathrm{DW}$ are taken from Salomon et al's 2015 publication $^{21}$ ) for 1 year equals 19\% of 'being dead for 1 year'. ${ }^{\text {iii }}$ However, if the YLL is merely a one-dimensional stretch of time, it does not yet embody any burden or disvalue which can be scaled. There is nothingcorresponding to disvalue-to calculate 19\% from. Surely, to be blind for 1 year is not the same as 'being dead' for $19 \%$ of 365 days, that is, 69.35 days. The DW must, rather, relate to the disvalue of 'being dead'. The YLD, on the other hand, is two-dimensional as it cannot exist independently of time and that-je ne sais quoi-without which the YLD would be time only.

Second, the antipodal objection is to maintain that the intended interpretation of the YLL is that its DW ' $D W_{\text {Death' }}$ ' is equal to $1-$ by definition. This defence is circular: the YLD is supposed to be defined in terms of the YLL and therefore cannot appeal to some value of being alive. Of course, our issue is not with the choice of 1 , but rather we question the assumption that any DW can be assigned to 'being dead' if YLLs is the foundational concept.

Where does this leave the current DALY? The burden of death is defined to be the number of years lost compared with an ideal lifespan. The burden of living with morbidity is morbidity's duration weighted by a comparison with living in full health. But recall that the yardstick for the DALY is the burden of death, not the other way around. The burden of blindness is supposedly not compared with not being blind, but rather how much less it burdens you than 'being dead'. The DALY is, therefore, not a gap-measure until one applies an account of how death burdens someone compared with being alive (in full health) compared with being blind (compared with being in full health). This seeming circularity is a problem for the DALY.

\section{INCOMMENSURABILITY}

The DALY model claims equivalence between YLLs and YLDs. The crucial question is if such equivalence makes sense? Whether or not it is meaningful to aggregate YLLs and YLDs into one summary number is partly a question of the commensurability of these quantities. There is a vast literature on commensurability. ${ }^{22-24}$ However, the question of whether the YLL and the YLD are, in fact, commensurable has received limited attention.

To begin with, two values are incommensurable when they lack a common measure. There are stronger and weaker types of incommensurability. Strong incommensurability occurs when something cannot be compared at all. One standard definition is that two values are strongly incommensurable when 'the one is neither more valuable than the other, nor less, and not of equal value' (Bradley et al, eds, p79). ${ }^{25}$ Strong incommensurability would apply to YLDs and YLLs if, as far as they are individual burdens, nothing can be said as to which is the lesser, nor if they

iii Thanks to an anonymous reviewer for making this point. 
are equal. It is no trivial matter to establish commensurability for YLLs and YLDs. After all, the YLL and the YLD seemingly lack a common yardstick, as individual YLLs do not appear to have a temporal extension for anyone.

A weaker type of incommensurability is called trumping or lexical orderings. The latter term derives from the fact that such orders resemble the alphabetical ordering of finite letter combinations. Lexical incommensurability does not deny that the incommensurable magnitudes are ordered or that they belong to the same class of entities but rather that the problem is to measure them in a common finite unit since they inhabit different 'tiers of importance'. Such orderings must, if they are to be represented numerically, be mapped to number systems which contain so-called non-standard numbers. ${ }^{\text {iv }}$

\section{THE COMMENSURABILITY OF THE YLD AND THE YLL}

We take the DALY to posit that YLDs and YLLs are commensurable. It appears more than reasonable to postulate that morbidity (and therefore YLDs) burdens the individual who embodies that morbidity. But accounting for how exactly death (understood as YLLs) burdens the deceased is demanding. To elaborate: the dying process burdens the living individual and is accounted for by the YLD. Note that when we talk about death, we do not mean this process of dying. By death, we refer to the instant in which someone goes from being alive to not living. We assume that death implies permanent non-existence, ${ }^{26}$ which makes 'being dead' an oxymoron. 'Being dead' would, if anything, mean the absence of any health state. It appears that the two constructs we are trying to relate via a common measure might not even belong to the same domain.

A viable avenue of inquiry should begin with the following question: Can death be bad for the individual who dies? If the answer is negative, we must conclude that YLLs cannot be an individual burden. This implies that the YLD and the YLL are incommensurable, as illustrated in figure 2. If the answer is affirmative, on some account $\mathrm{X}$, we can proceed to the next question: Does X provide commensurability between the YLD and the YLL?

There are several arguments against the view that death is bad for those who die, and the three strongest of them concern experience, time and symmetry. According to the experience argument, burdens are something we must experience. But, we cannot experience while 'being dead'. Thus, death cannot be bad for us. According to the time argument, most burdens begin at a time and are bad for us through time but there is no time when death is bad for us. When we are alive, we have not lost our lives, and when we 'are dead', we do not exist. Thereforethe argument goes-death cannot be bad for us. ${ }^{v}$ According to the symmetry argument, we do not consider the non-existence before our lives as a burden, so why should the non-existence after ${ }^{\text {vi }}$ our lives-death-be one ${ }^{27}$ Any of these three Epicurean arguments makes it questionable whether the YLL quantifies an individual burden at all. ${ }^{\text {vii }}$

\footnotetext{
${ }^{\text {iv }}$ Non-Archimedean number systems, employing so-called infinitesimals and infinite numbers, are readily available but they are unfit for standard health economic analysis.

${ }^{\mathrm{v}}$ The two first arguments are rooted in Epicurus. ${ }^{48}$

${ }^{\mathrm{vi}}$ This latter argument is rooted in Lucretius. ${ }^{27}$

vii Note that the problem is not one of terminology. We do not believe YLLs are mislabelled as a burden but that they are, in fact, incommensurable with YLDs.
}

Table 1 The years lived with disability (YLDs) and the years of life lost (YLLs)

\begin{tabular}{llll}
\hline & YLD & YLL \\
\cline { 2 - 2 } Dimensions & $\begin{array}{l}\text { Two-dimensional } \\
\text { (timexdisability weight) }\end{array}$ & $\begin{array}{l}\text { One-dimensional } \\
\text { (time) }\end{array}$ \\
\hline $\begin{array}{l}\text { Concern about individual burden } \\
\text { Experience: Do we experience it? }\end{array}$ & Yes & No \\
$\begin{array}{l}\text { Time: Does it harm us through } \\
\text { time? }\end{array}$ & Yes & No \\
$\begin{array}{l}\text { Symmetry: Does it treat the past } \\
\text { and future equally? }\end{array}$ & Yes & No \\
\hline
\end{tabular}

Based on the three arguments, we may question whether YLLs measure an individual burden, if not, then YLDs and YLLs will be incommensurable.

Notice how the YLD is unaffected by these arguments. First, morbidity is usually something that people experience. Second, morbidity happens at a time and is harmful through time. Third, it is not unreasonable that we count people's past and future morbidity as equally bad in the GBD. Accordingly, there are several significant asymmetries between the YLD and the YLL, which we have summarised in table 1.

Two things being different in important aspects do not, in and of itself, imply their incommensurability. But if these three arguments are correct, then the YLL cannot be an individual burden and so YLDs and YLLs are incommensurable qua individual burdens.

In brief, the above poses two foundational problems for the DALY. First, we must provide a reasonable account of the burden associated with YLLs that overcomes the three Epicurean arguments. Second, this account must give rise to a notion of burden that makes YLLs commensurable with YLDs.

\section{THREE RESPONSES}

We can think of at least three responses to this incommensurability problem—ignore, accept or rescue (see figure 3).

\section{Ignore the problem}

The first response is simply to ignore the problem; the DALY is a convenient tool for measuring unhealth and sufficiently good to the extent that the public accepts it. This answer is unsatisfactory. The GBD study is supposed to provide evidence to inform public policy. ${ }^{8}$ A burden is something that we should get rid of, it demands action and it is too imperative to be measured incorrectly. Consequently, we reject this response as adequate.

\section{Accept the incommensurability}

The second response is to accept that YLDs and YLLs are, in fact, incommensurable and refrain from aggregating them into DALYs. There is nothing inherently wrong in reporting YLDs alongside YLLs, and allow decision-makers to act on this information as they see fit, but the splitting strategy is not an easy solution either. This destroys the DALY's raison d'etre of obtaining an integrated measure of morbidity and mortality, and it yields two competing measures of burden, which would imply that we de facto abandon the DALY model.

Given the purpose of the DALY model as a resource in policy-making, ${ }^{8}$ we will need to know how to trade-off YLDs and YLLs. Given strong incommensurability, there is certainly no answer to this trade-off if any number of YLDs is not worse, better or equal to any number of YLLs. Given the weak incommensurability, it is, moreover, not satisfactory if YLLs always trump YLDs or vice versa. Under either type of incommensurability, policy 
based on YLDs and YLLs will not make much sense. Hence, we discard this position.

\section{Rescue}

The third option is to rescue their commensurability by one of two strategies: (3.1) redefine the YLD and the YLL so that they no longer concern individual burden, thereby being commensurable for something else or (3.2) argue that the YLD and the YLL are, in fact, commensurable for individual burden.

(3.1) Redefining can be done in at least two ways. First, (3.1.1) we could consider the DALY as a measure of 'population burden'. On such a queer view, the burden belongs to the population proper rather than the individual proper, and so avoids the Epicurean arguments, as the population remains while individuals enter and leave. To our knowledge, no one has hitherto offered an adequate account of population burden in this sense. It is reasonable to believe that what matters is that disability is a burden for individual human beings. If the burden we measure does not burden $u s$, we fail to see why we should care about it. Moreover, such a view departs from the stated aims of the DALY. For these reasons, we discard this position.

A second strategy (3.1.2) is to redefine the DALY as a measure of societal burden. Societal burden, in this context, refers to everyone who is indirectly affected by the disease burden in question. This strategy has a certain pull to it as premature death clearly affects family, friends and the wider society. Such a strategy could take several forms. One could adopt a pure societal burden-account, and the canonical choice would then be to measure the burden in pecuniary terms: for example, a willingness-to-pay measure. This is abdicating from the task of the DALY. $^{15}$

Another way is adopting a combined account of individual and societal burden. For instance, by claiming that the YLL is a type of individual burden which is nevertheless distinct from the type of individual burden measured by the YLD-i.e. the YLL as a burden on individuals other than the deceased. To such an account, the three Epicurean arguments do not apply. In contrast to the population burden-account, the burden is now placed on other ephemeral individuals rather than on the permanent population. There are two challenges with this latter approach. For one, this combined strategy implies that YLDs and YLLs will never belong to the same individual. Hence, it makes less sense to monitor inequalities in health by DALYs. For another, there is a slippery slope in this strategy. It is hard to see how we can maintain that Alice's death would burden Beth, but Alice's broken leg would not. Pursuing such an avenue, it seems, would lead us to a 'gross domestic product (GDP) of burdens' rather than a metric for individual burden. This would directly contradict the egalitarian ${ }^{\text {viii }}$ aim of the GBD. ${ }^{8}$

Strategy (3.2) is, we will argue, more promising. We know that YLDs quantifies unhealth; an inherently multidimensional construct. More precisely, in GBD 2015, the YLD quantifies the burden of 235 distinct conditions. In other words, it is assumed that these conditions are commensurable as individual burdens. However, several of these conditions are not intuitively commensurable for a common value. Compare, for instance, mild low back pain, moderate hearing loss, amputation of one arm with treatment or severe dementia. ${ }^{21}$ The question is: What is the unifying attribute common in these otherwise

\footnotetext{
viii That is, the egalitarian standard of measurement as opposed to an egal-
} itarian distribution of the measured burden. quite different conditions that make them commensurable qua burden? To answer this, we will need an account of harm, to begin with.

Conditions measured in the GBD categorised as musculoskeletal disorders such as low back pain or neck pain are intuitively harmful because they involve a continuous experience of pain. On the other hand, conditions like complete hearing loss and vision loss are not primarily harmful because of what you experience but rather because of what you do not experience. Moreover, amputation of limbs is harmful because the loss of the limb can incur a significant opportunity loss for the individuals in question. ${ }^{\text {ix }}$ These latter examples thus clearly presume a counterfactual account of harm.

How does this account explain the burden of the YLL? Given our assumption that death implies permanent extinction, death most certainly is nothing like experiencing pleasure or pain. Death cannot harm us in an absolute or robust sense. In the philosophical literature, the standard account is that death harms those who die in a comparative sense only.

To clarify, note that we are not alluding to any particular theory. Rather, we are aiming to appeal to some theory which can adequately account for how Epicurus-during his existence-could have been harmed by his death by an appeal to how his life could counterfactually have ended had he not died when he did.

A counterfactual account of harm seems capable of countering the experience argument: something can be bad for you, counterfactually, even if inexperienced. With regard to symmetry, if death is counterfactually bad for us then why cannot the beginning of our existence be counterfactually bad for us? We do not regard the symmetry argument as a strong one. While our deaths lie in an open future, our pre-existence lies in a fixated past. It seems reasonable that counterfactual harm should be directed at an open future. In brief, while the beginning of an individual's existence is a highly elusive concept, we maintain that harm happens to existing individuals and that counterfactual harm is connected to one's counterfactual futures, and not one's counterfactual pasts.

We are then left with the timing of death's badness. Some may argue that the foundational question is whether a deceased person can have a well-being level. According to one position-subsequentism-your well-being level is reduced to zero at all times when you counterfactually could have been alive. $^{2528}$ This account, if correct, provides a yes to the question of whether death can be an individual burden. This theory also seems to accomplish the second task by being a theory $\mathrm{X}$ which gives credibility to commensurability. There are problems with subsequentism that we do not discuss here. ${ }^{29} 30$

What is the status of a person's well-being-determining or health-determining properties? ${ }^{\mathrm{X}}$ If there is no such correlate, does this mean that we must abandon the DALY?

There may be alternative views for death's badness-that unifies the YLD and the YLL-and that avoids appealing to well-being levels for 'being dead'. While inquiry into such wellbeing levels is indeed fruitful-since they would likely suffice for commensurability-we do not believe that such well-being levels are necessary. The main difference between accepting and rejecting well-being levels for 'being dead' is that accepting them allows constructing YLDs from YLLs (this is the current DALY model). While rejecting such levels likely leaves constructing

${ }^{i x}$ Phantom pain is not the characteristic feature of amputated limbs.

${ }^{\mathrm{x}}$ Thanks to an anonymous reviewer for raising this question. 
YLLs from YLDs the only viable option. This latter strategy would amount to a push in the direction of actually making DALYs inverse QALYs. All these strategies seem to bring a notion of well-being into the DALY framework which is counter to the stated aims of the GBD. ${ }^{2 \times i}$

There are several possible views for the timing or non-timing of death's badness, ${ }^{\mathrm{xii}}$ and challenges of forecasting the relevant counterfactual scenarios that we do not discuss here. That being said, a counterfactual notion of harm seems to be the most viable option for making the YLD and the YLL commensurable. As a starting point, death is bad (or good) compared with the life you could have had, had you not died. ${ }^{\text {xiii }}$ This means that reference to counterfactual scenarios can help explain how death harms us. $^{25} 262831-34$

We recognise that the DALY is often referred to as a gap-measure. ${ }^{35-37}$ In this sense, it relies on counterfactual thinking. But note that YLLs and YLDs separately are motivated counterfactually as gaps to two-although different-ideals. First, a condition's DW is the gap to ideal health, and second, the YLL is the gap between the time of death and ideal longevity. But this does not ensure their commensurability. Our proposal is that in order for YLDs and YLLs to be commensurable for individual burden, one must rely on a counterfactual account of harm.

It is because death harms us in a comparative sense that death (and therefore YLLs) is an individual burden. For that reason, whatever is measured by YLLs and YLDs have something important in common: the counterfactual harm. In this way, YLDs and YLLs may become commensurable. If accepted, this strategy will allow us to speak of DALYs as a pure measure of individual burden.

This strategy will come at a cost for the GBD authors: they will need to take a stance on which counterfactual account of the harm of death that is the correct one.

In the current GBD, around 2.7 million annual neonatal deaths are counted. The major cause of these deaths is prematurity. Moreover, each of these deaths is counted as the greatest possible burden (approximately 86 DALYs). On the other hand, there are around 2.6 million annual stillbirths, ${ }^{\text {xiv38 }}$ and these deaths do not contribute to the DALY count. Stillbirths have recently been estimated and monitored ${ }^{39}$ but do not presently ${ }^{40}$ generate YLLs. ${ }^{\mathrm{xv}}$ Additionally, we can reasonably ask why a death just before birth is not at all harmful (ie, not bad in a comparative sense), while a death just after birth is the worst possible harm. ${ }^{\text {xi }}$ If a counterfactual account of harm is to underlie the YLL, this will involve a re-examination of the age at which we should begin counting YLLs and at which age death should incur the greatest number of YLLs. We argue that the issue of which individuals that can generate DALYs should be informed by the answer to

\footnotetext{
${ }^{x i}$ Broome (unpublished manuscript) furthers an argument for why the DALY should measure well-being rather than health.

xii In addition to subsequentism, the other views that reject the time argument are priorism, concurrentism, atemporalism, eternalism and indefinitism. Among these views, subsequentism, atemporalism and priorism are most popular. ${ }^{30}$

xiiiThere are several technical expositions of counterfactual harm accounts $^{25} 28{ }^{31-33}$ but we do not want to commit to any particular version here.

${ }^{\text {xiv }}$ That is, WHO definition of antenatal fatality between 28th week of gestation and birth.

${ }^{\mathrm{xv}}$ Even though there is a considerable overlap in conditions that incur stillbirths and premature neonatal deaths, for example, sepsis and encephalopathy.

${ }^{x v i}$ As the GBD authors note, an estimation of fetal deaths is an important
} area of future work (Murray et al, p8). ${ }^{2}$ the following question: Which individuals can be counterfactually harmed by their death? We remain agnostic to what age one should begin the DALY count and refer to the growing literature on this critical issue for further guidance. ${ }^{40-44}$

\section{CONCLUSION}

In this paper, we have addressed one critical objection to the DALY model. We have argued that YLLs and YLDs are, in fact, incommensurable unless the individual burden of the YLL is explained. Neglecting this problem is not an option. Moreover, we offer a solution to this problem: a counterfactual account of harm. However, this counterfactual strategy implies that we need to readdress the question of at what age and time death can be counterfactually harmful to the individual.

Acknowledgements Thanks to the audiences at these meetings for their constructive comments. We are especially grateful to John Broome, Lars Christie, Espen Gamlund, Olav Gjelsvik, Pål Gulbrandsen, Maximillian Kiener, Ole Koksvik, Reidar K Lie, Joseph Millum, Karl Erik Müller, Joshua Shepherd, Preben Sørheim, Hilde Vinje, Dominic Wilkinson and two anonymous reviewers for their valuable comments on earlier drafts of this article. This work was presented at Ahus University College, the Global Health Priorities' Finse Seminar, the University of Bergen, University of Birmingham, University of Oslo and University of Oxford.

Contributors CTS had the initial idea, with input from OFN and MB. CTS and $M B$ drafted the manuscript, and OFN revised it along the way. The three authors regularly met for work sessions. MB drafted the figures and tables, and CTS made revisions. All authors subsequently read, revised and approved the final manuscript.

Competing interests None declared.

Provenance and peer review Not commissioned; externally peer reviewed.

Open Access This is an Open Access article distributed in accordance with the Creative Commons Attribution Non Commercial (CC BY-NC 4.0) license, which permits others to distribute, remix, adapt, build upon this work non-commercially, and license their derivative works on different terms, provided the original work is properly cited and the use is non-commercial. See: http://creativecommons.org/ licenses/by-nc/4.0/

(C) Article author(s) (or their employer(s) unless otherwise stated in the text of the article) 2018. All rights reserved. No commercial use is permitted unless otherwise expressly granted.

\section{REFERENCES}

1 Murray CJ, Ezzati M, Flaxman AD, et al. GBD 2010: design, definitions, and metrics. Lancet 2012:380:2063-6.

2 Murray CJ, Ezzati M, Flaxman AD, et al. Comprehensive systematic analysis of global epidemiology: definitions, methods, simplification of DALYs, and comparative results from the global burden of disease study 2010. Lancet 2012;380:1-140.

3 Horton R. GBD 2010: understanding disease, injury, and risk. The Lancet 2012;380:2053-4.

4 Dempsey M. The death registration states. Am Rev Tuberc 1948;57:306

5 Haenszel W. A standardized rate for mortality defined in units of lost years of life. Am J Public Health Nations Health 1950:40:17-26.

6 Murray CJ, Lopez AD. Measuring the global burden of disease. N Engl J Med 2013;369:448-57

7 The World Bank. World development report 1993- Investing in health. New York, USA Oxford University Press, 1993. http://bases.bireme.br/cgi-bin/wxislind.exe/iah/online/? IsisScript=iah/iah. $x$ is\&src=google\&base=REPIDISCA\&lang $=p \&$ nextAction $=$ Ink\& exprSearch $=63448 \&$ indexSearch $=I D$ (accessed 5 Dec 2016).

8 Murray CJL, Acharya AK. Understanding DALYs. J Health Econ 1997;16:703-30.

9 Lyttkens CH. Time to disable DALYs? On the use of disability-adjusted life-years in health policy. Eur J Health Econ 2003;4:195-202.

10 Arnesen T, Kapiriri L. Can the value choices in DALYs influence global priority-setting? Health Policy 2004:70:137-49.

11 Anand S, Hanson K. Disability-adjusted life years: a critical review. J Health Econ 1997:16:685-702.

12 Schroeder SA. Value choices in summary measures of population health. Public Health Ethics 2016:phw032.

13 Sullivan DF. A single index of mortality and morbidity. HSMHA Health Rep 1971:86:347-54.

14 Torrance GW, Thomas WH, Sackett DL. A utility maximization model for evaluation of health care programs. Health Serv Res 1972:7:118-33.

15 Murray CJ. Quantifying the burden of disease: the technical basis for disabilityadjusted life years. Bull World Health Organ 1994;72:429-45. 
16 Weinstein MC, Torrance G, McGuire A. QALYs: the basics. Value Health 2009:12:S5-S9.

17 Hofstetter P, Hammitt JK. Selecting human health metrics for environmental decisionsupport tools. Risk Anal 2002;22:965-83.

18 Murray CJL, Salomon JA, Mathers CD, et al. Summary measures of population health : concepts, ethics, measurement and applications. SMPH, 2002. http://www.who.int/ iris/handle/10665/42439 (accessed 5 Dec 2016).

19 Robberstad B. QALYs vs DALYs vs LYs gained: What arethe differences, and what difference do they make for health carepriority setting? Nor Epidemiol 2009;15.

20 Miyamoto JM, Wakker PP, Bleichrodt $\mathrm{H}$, et al. The zero-condition: a simplifying assumption in QALY measurement and multiattribute utility. Manage SCi 1998:44:839-49.

21 Salomon JA, Haagsma JA, Davis A, et al. Disability weights for the Global Burden of Disease 2013 study. Lancet Glob Health 2015;3:e712-e723.

22 Chang R. Incommensurability, incomparability, and practical reason. Cambridge: Harvard University Press, 1997.https://www.google.com/books?hl=no\&lr=\&id=yWB Wel4VgJgC\&oi=fnd\&pg=PR9\&dq=Incommensurability, + Incomparability, + and + Prac tical+Reason.+chang\&ots $=C Y L p N 95 y 0 t \& s i g=b R 8 c T V j c D I 3 P h k F P X L W Z k Y 7 c$ tk

23 Griffin J. Well-Being: its meaning, measurement, and moral importance. Oxfordshire, Oxford: Clarendon Press, 1989.

24 Hsieh N. Incomensurable values. Zalta EN, ed. Standford encyclopedia of philosophy: Spring, 2016. http://plato.stanford.edu/archives/spr2016/entries/valueincommensurable/ (accessed 13 Sep 2016).

25 In: Bradley B, Feldman F, Johansson J, eds. The Oxford handbook of philosophy of death. Oxford, New York: Oxford University Press, 2013.

26 Nagel T. Death. Noûs;1970:73-80.

27 L. On the nature of the universe: Penguin Books, 1951. https://ia801604.us.archive. org/23/items/in.ernet.dli.2015.53892/2015.53892.Lucretius-On-The-Nature-Of-TheUniverse-1951.pdf (accessed 11 Jul 2017).

28 Feit N, Harm C. Creation and death. Utilitas 2016;28:136-63.

29 Silverstein $\mathrm{H}$. The time of the evil of death. In: Campbell JK, O'Rourke M, Silverstein $\mathrm{H}$, eds. Time and identity. Cambridge: MIT Press, 2010:283-95.

30 Johansson J. The timing problem. The oxford handbook of philosophy of death. New York, USA: Oxford University Press, 2012:255-73.

31 Klocksiem J. A defense of the counterfactual comparative account of harm. Am Philos Q 2012;49:285-300
32 Purves D. Accounting for the harm of death. Pac Philos Q 2016;97:89-112.

33 Broome J. Weighing lives: Oxford University Press, 2006. https://ideas.repec.org/b/ oxp/obooks/9780199297702.html (accessed 5 Dec 2016).

34 Feldman F. Some puzzles about the evil of death. Philos Rev 1991;100:205-27.

35 Murray C, DALYs R. The global burden of disease: a comprehensive assessment of mortality and disability from diseases, injuries, and risk factors in 1990 and projected to 2010. Geneva: Harvard School of Public Health, 1996:50-98.

36 Murray CJ, Salomon JA, Mathers C. A critical examination of summary measures of population health. Bull World Health Organ 2000;78:981-94.

37 Murray CJL, Evans DB. Health systems performance assessment: debates, methods and empiricism: World Health Organization, 2003.

38 Lawn JE, Blencowe H, Pattinson R, et al. Stillbirths: Where? When? Why? How to make the data count? The Lancet 2011:377:1448-63.

39 GBD 2015 Child Mortality Collaborators. Global, regional, national, and selected subnational levels of stillbirths, neonatal, infant, and under-5 mortality, 19802015: a systematic analysis for the Global Burden of Disease Study 2015. Lancet 2016;388:1725-74.

40 Phillips J, Millum J. Valuing Stillbirths. Bioethics 2015;29:413-23.

41 Jamison DT, Shahid-Salles SA, Jamison J. et a/Incorporating deaths near the time of birth into estimates of the Global Burden of Disease. In: Lopez AD, Mathers CD, Ezzati M, Jamison DT, Murray CJ, . eds. Global burden of disease and risk factors. Washington (DC): World Bank, 2006. http://www.ncbi.nlm.nih.gov/books/ NBK11805/(accessed 5 Dec 2016).

42 Millum J. Age and death: a defence of gradualism. Utilitas 2015;27:279-97.

43 Solberg CT, Gamlund E. The badness of death and priorities in health. BMC Med Ethics 2016:17:21.

44 In: Solberg CT, Gamlund E, eds. Saving people from the badness of death. New York USA: Oxford University Press.

45 Schroeder SA. Incidence, prevalence, and hybrid approaches to calculating disabilityadjusted life years. Popul Health Metr 2012;10:19.

46 Voigt K, King NB. Disability weights in the global burden of disease 2010 study: two steps forward, one step back? Bull World Health Organ 2014;92:226-8.

47 Hausman DM. Health, well-being, and measuring the burden of disease. Popul Health Metr 2012:10:13

48 Oates WJ. The Stoic and epicurean philosophers the complete extant writings of epicurus, epictetus, lucretius [and] marcus aurelius: Random House, 1940. 